Sherif Beniameen Mossad, MD, FACP, FIDSA, FAST

Department of Infectious Diseases, Respiratory Institute, and Transplant

Center, Cleveland Clinic; Professor of Medicine, Cleveland Clinic Lerner

College of Medicine of Case Western Reserve University, Cleveland, $\mathrm{OH}$

\title{
Fungus among us: A poster child for diagnostic stewardship
}

$I^{\mathrm{n}}$ $\mathrm{n}$ this issue of Cleveland Clinic Journal of Medicine, El-Baba et al address the clinical significance of Aspergillus species isolated from respiratory cultures. ${ }^{1}$ The authors elegantly and succinctly summarize the clinical classification and diagnostic approach to Aspergillusrelated lung disease.

See related article, page 543

Interpretation of diagnostic studies, including microbiologic tests, should always be predicated on the clinical indication for testing. ${ }^{2}$ So before interpreting the clinical significance of isolation of Aspergillus species from respiratory cultures, we should first ask whether the culture was clinically indicated, or whether this was an incidental finding.

Aspergillus species are ubiquitous in the water environments of the home and of healthcare facilities. ${ }^{3}$ Therefore, while Aspergillus species can cause several forms of lung disease, some of which are life-threatening, incidental growth of this organism should be expected due to contamination or colonization. Contamination refers to the transient presence of this organism in the airways without causing illness, or its accidental addition to inanimate objects in the process of collection, transport, or processing in the laboratory. Colonization refers to the persistent presence of the organism in the airways, again without causing illness, but it can be one step away from resulting in clinical disease. These concepts apply to other human organ systems, including the skin and the urinary tract. ${ }^{4}$

\section{EVOLVING DEFINITIONS OF FUNGAL INFECTIONS}

Clinicians have struggled to define fungal infections at the bedside for several decades. The first international consensus defining opportunistic invasive fungal infections in immunocompromised patients with cancer and hematopoietic stem cell transplants was published in 2002. ${ }^{5}$ Diagnostic and management approaches to invasive fungal infections evolved rapidly over the last 2 decades, necessitating consecutive updates in $2008^{6}$ and $2020 .^{7}$

These consensus definitions were intended to harmonize research studies but nevertheless have been widely adopted for clinical practice. With each update, the definition of the "probable" category expanded, while the scope of the category "possible" was contracted. The International Society for Heart and Lung Transplantation published its own standardized definitions pertaining to lung and heart transplant recipients. ${ }^{8}$

\section{NEEDED: DIAGNOSTIC STEWARDSHIP}

El-Baba et $\mathrm{al}^{1}$ describe the diagnostic accuracy of the available imaging and laboratory tests, their limitations, and the risks associated with invasive bronchoscopic and surgical procedures necessary for histopathologic confirmation.

Our antifungal drug options are limited, and most agents have significant adverse effects and drug interactions and are expensive, further complicating management decisions. Practice guidelines by the Infectious Diseases Society of America, ${ }^{9}$ the American Society of Transplantation, ${ }^{10}$ and the American Society of Transplantation and Cellular Therapy ${ }^{11}$ provide excellent guidance in these patient populations.
Interpretation of diagnostic studies, including microbiologic tests, should always be predicated on the clinical indication for testing 
If all patients in whom Aspergillus species grow from respiratory cultures were to be treated, the risks would outweigh the benefits. In making these decisions, clinicians should apply the principles of diagnostic stewardship ${ }^{2}$ before applying the principles of antimicrobial stewardship. ${ }^{12}$
El-Baba et al provide a clinically driven, systematic approach to applying these principles.

\section{DISCLOSURES}

The author reports no relevant financial relationships which, in the context of his contributions, could be perceived as a potential conflict of interest.

\section{REFERENCES}

1. El-Baba F, Watza D, Soubani AO. Is Aspergillus isolated from respiratory cultures clinically significant? Cleve Clin J Med 2021; 86(0):543546. doi:10.3949/ccjm.88a.20188

2. Miller JM, Binnicker MJ, Campbell S, et al. A guide to utilization of the microbiology laboratory for diagnosis of infectious diseases: 2018 Update by the Infectious Diseases Society of America and the American Society for Microbiology. Clin Infect Dis 2018; 67(6):e1e94. doi:10.1093/cid/ciy381

3. Richardson M, Rautemaa-Richardson R. Exposure to Aspergillus in home and healthcare facilities' water environments: focus on biofilms. Microorganisms 2019; 7(1):7. doi:10.3390/microorganisms7010007

4. Dani A. Colonization and infection. Cent European J Urol 2014; 67(1):86-87. doi:10.5173/ceju.2014.01.art19

5. Ascioglu S, Rex JH, de Pauw B, et al; Invasive Fungal Infections Cooperative Group of the European Organization for Research and Treatment of Cancer and Mycoses Study Group of the National Institute of Allergy and Infectious Diseases. Defining opportunistic invasive fungal infections in immunocompromised patients with cancer and hematopoietic stem cell transplants: an international consensus. Clin Infect Dis 2002; 34(1):7-14. doi:10.1086/323335

6. De Pauw B, Walsh TJ, Donnelly JP, et al; European Organization for Research and Treatment of Cancer/Invasive Fungal Infections Cooperative Group; National Institute of Allergy and Infectious Diseases Mycoses Study Group (EORTC/MSG) Consensus Group. Revised definitions of invasive fungal disease from the European Organization for Research and Treatment of Cancer/Invasive Fungal Infections Cooperative Group and the National Institute of Allergy and Infectious Diseases Mycoses Study Group (EORTC/MSG) Consensus Group. Clin Infect Dis 2008; 46(12):1813-1821. doi:10.1086/588660

7. Donnelly JP, Chen SC, Kauffman CA, et al. Revision and update of the consensus definitions of invasive fungal disease from the European Organization for Research and Treatment of Cancer and the Mycoses Study Group Education and Research Consortium. Clin Infect Dis 2020; 71(6):1367-1376. doi:10.1093/cid/ciz1008

8. Husain S, Mooney ML, Danziger-Isakov L, et al; ISHLT Infectious Diseases Council Working Group on Definitions. A 2010 working formulation for the standardization of definitions of infections in cardiothoracic transplant recipients. J Heart Lung Transplant 2011; 30(4):361-374. doi:10.1016/j.healun.2011.01.701

9. Patterson TF, Thompson GR III, Denning DW, et al. Practice guidelines for the diagnosis and management of aspergillosis: 2016 update by the Infectious Diseases Society of America. Clin Infect Dis 2016; 63(4):e1-e60. doi:10.1093/cid/ciw326

10. Husain, S, Camargo, JF; on behalf of the AST Infectious Diseases Community of Practice. Invasive aspergillosis in solid-organ transplant recipients: guidelines from the American Society of Transplantation Infectious Diseases Community of Practice. Clin Transplant 2019; 33:e13544. doi:10.1111/ctr.13544

11. Sanjeet S, Dadwal, Tobias M, et al. American Society of Transplantation and Cellular Therapy Series. 2: Management and prevention of aspergillosis in hematopoietic cell transplantation recipients. Transplantation and Cellular Therapy 2021; 27(3):201-211. doi:10.1016/j.jtct.2020.10.003

12. Dellit TH, Owens RC, McGowan JE, et al. Infectious Diseases Society of America and the Society for Healthcare Epidemiology of America guidelines for developing an institutional program to enhance antimicrobial stewardship. Clin Infect Dis 2007; 44(2):159177. doi:10.1086/510393

Address: Sherif Beniameen Mossad, MD, FACP, FIDSA, FAST, Department of Infectious Diseases, G21, Cleveland Clinic, 9500 Euclid Avenue, Cleveland, $\mathrm{OH} 44195 ;$ mossads@ccf.org 